International Review of Research in Open and Distributed Learning Volume 20, Number 5

December - 2019

\title{
A Survey of OER Implementations in 13 Higher Education Institutions
}

Rory McGreal

Research Fellow at Contact North/Contact Nord, Athabasca University

Institutions in many jurisdictions are in the process of implementing Open Educational Resources (OER). This short paper is based on a report commissioned by Contact North/Contact Nord as part of their Pockets of Innovation series to better understand the impact of OER implementations at diverse institutions.

The investigation looks into 13 different OER implementations at the postsecondary level: three community colleges and one university. Four are in the United States; three universities and one Indigenous college in Canada; and five international universities-in Africa, the Middle East, South America, and Southeast Asiai.

The format of the investigation followed a standard five-point inquiry model:

1. Opportunity: Why did they decide to implement OER?

2. Innovation: What did they do?

3. Benefits: What were the outcomes of the intervention?

4. Challenges: What were the problems in the implementation?

5. Potential: How do they see the future of the project?

The data collected consisted of researching relevant papers and soliciting opinions from lead participants in OER implementations. All information was taken directly from telephone interviews with a local reporter or by studying the documentation that was available either in articles or on websites. Points not stated by these reporters were not necessarily missing from their implementation, but they were not highlighted in the reports. The only consensus found among the different implementations was on the cost savings OER provided for students and/or the administration, with no other generally agreed upon points in the five areas of inquiry. As well, there were no other consensus points among the institutions in Canada, the United States, and the five international institutions. 
Cost savings for students was the only agreed upon consensus (12/13 institutions). The lone outlier was an institution (Athabasca University in Canada) that included course material costs in the tuition. This institution reported on significant savings for the institution. Seven other institutions also reported cost savings over and above those of the students.

\section{Opportunity}

For a small majority (7) of the institutions, the OER opportunity was catalysed by an external grant. Seven institutions also mentioned quality assurance as a major reason for deciding to implement OER, and, for seven implementing institutions, teaching effectiveness was reported as being an important factor in their decision. Some institutions mentioned more than one of these factors. Three institutions identified the following reasons: Their desire to promote innovation among the staff; aspiring for leadership in technology; encouraging both internal and external collaboration among faculty; using OER to more closely align the content with the curriculum; reducing development time; student retention; and student access. Surprisingly to this author, only one institution mentioned the desire to design a MOOC (Massive Open Online Course). This could be because the main priority of most of the institutions researched was for classroom-based and blended learning, with little interest in fully online courses.

\section{Innovation}

A significant number (6) of institutions reported that their innovation was in building a culture of openness among faculty, while several (4) others highlighted the creation of a content repository as their innovation. A few (3) noted that simply introducing new technologies was the most important innovation for their institutions. Several highlighted the creation of a content repository as their innovation. A smaller number (3) of institutions made the following points: That the integration of OER into their courses was the most innovative result; adding ancillary content was considered progressive; faculty became innovative in their pedagogy; faculty and librarians collaborated for the first time; and implementing OER raised the institutional reputation for innovation in their communities.

\section{Benefit}

Most institutions (8) felt that the primary benefit for faculty was the sharing of resources (both internal and external) and collaborations. Many (6) reported on significant improvements in both content and teaching quality, noting that OER gave them the legal, technical, and pedagogical flexibility that they needed to localise, update, or otherwise improve content and their teaching. Legally, the permission to alter the content was considered to be an important improvement over the commercial content that they could not change when needed. Technically, the OER could be ported to any device and pedagogy was improved when instructors could decide on the content and fit it to their teaching styles. Other benefits for institutions mentioned (4) include using OER to introduce new pedagogies, reducing the time needed for course development, and the ability to adapt and change the content to suit their teaching. Some institutions mentioned the ability to update their courses and use technology effectively. Other institutions noted that implementing OER left them with increased time for research as OER saved them significant preparation time and in addition, this resulted in their increased recognition as innovators. 
Student benefits focused on the significant cost savings as mentioned above. Several (5) felt that improvements in content quality and more innovative teaching combined with the easy access provided by OER that could be ported to different devices was an important benefit. Some (3-) commented that because of the cost-savings of OER, students were able to successfully remain in their courses.

Institutional benefits mentioned included the cost savings and the retention of students (which translates into increased revenues). The OER implementation also led to open policies improving the working environment for faculty and administration.

\section{Challenges}

As a major challenge, while some institutions reported reduced workload due to OER implementation, others (5) suffered from work overload, primarily associated with the time needed to assess the quality of imported OER. The lack of technical expertise by faculty was also a significant challenge; as was the lack of understanding of the legal issues around copyright. Also, some faculty felt it was time consuming to search out, find, and then adapt imported OER to their requirements. The low bandwidth (or none) available at home was considered a major obstacle for students. This problem was identified in the international institutions and not mentioned in the North American ones. Some felt that there was excessive content in the OER courses. Faculty reported difficulties in citing OER, while others reported difficulties in printing out the content when needed.

A major challenge for institutions was building faculty awareness of OER. They also reported on problems related to the poor infrastructure available that caused serious problems in implementation.

\section{Potential}

There were a wide variety of responses regarding the potential for OER at their institution. They reported on the potential for more cost savings and a more active faculty. They felt that OER would help them to attract and retain students, but strategies and plans needed to be developed.

One university reported on using OER to support a public - private partnership with a company that specialized in testing, while another such partnership was created between community college and a private company that aided in the actual implementation of the OER. There was one indigenous community college that became very excited about OER and their ability to adapt the content to address their unique concerns.

These 13 implementations have provided important information of use to any institutions considering OER initiatives in the future. Each institution identified specific opportunities, challenges, etc. Other than the cost-savings, there was no consensus on any other issues. While access to a grant was important for the majority of institutions, almost half chose to proceed with their OER initiative with no external support. Nearly half the institutions surveyed identified that a principal driver for innovation in their institution was the common desire for a culture of openness. This points to the need for faculty awareness in the possibilities of OER and other aspects of open learning. 


\section{Discussion}

In addressing the high cost of course content, while supporting a culture of collaboration, the majority of institutions recognized the need for sharing resources among faculty. Thus, they promoted the OER initiative as a means of supporting sharing and teamwork among faculty, both within and outside the institution. Several institutions reported on a reduced workload, while several others complained that their workload increased. This phenomenon could be ascribed to the approach that the different institutions took towards implementation. If an institution searches and finds a course textbook or a large module and adopts it "as is," this reduces the workload. These faculty may not be too concerned with the content being an exact match, while those who reported an increased workload, either created their own materials or spent much time in adapting, integrating, and localising the OER chosen. Whatever the path chosen, it is evident that the OER movement is not simply about free content. The affordances of OER, across different cultures, includes many benefits, such as supporting learning design, a more collaborative faculty, and increasing student retention

The case studies form part of Contact North/ContactNord's Pockets of Innovation series. These 13 examples provide insights into multiple facets of OER development, use, and impact:

- Use of OER for teaching and learning;

- Integration of OER into programs and courses;

- Development of OER by individual faculty, as well as teams;

- Adaptation of OER to match culture, language, and course content requirements;

- Strategies to involve faculty in the development and use of OER;

- Benefits of OER for student learning and faculty effectiveness; and

- Organizational change resulting from the integration of OER.

The descriptions outline the organizational contexts that motivated the introduction of OER into teaching and learning, as well as details of the policies, practices, development processes, and resources involved. Benefits and challenges for faculty, students, and, in some examples, institutions, receive particular attention, followed by consideration of the potential for OER use in each academic situation.

Contacts from each institution are provided for each description with links to further information. 


\section{Athabasca}

University

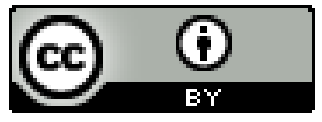

${ }^{\mathrm{i}}$ https:// teachonline.ca/ tools-trends/ open-education-resources-oer-applications-around-world/ taxonomy-term 